\title{
THE INFLUENCE OF BANDWIDTH AND TEMPORAL PROPERTIES OF SPATIAL NOISE ON BINOCULAR MASKING-LEVEL DIFFERENCES ${ }^{1}$
}

\author{
G. Bruce Henning \\ Department of Experimental Psychology, University of Oxford, OX1 3UD, England \\ and
}

B. Gevene Hertz

The Biomedical Engineering Center, Technological Institute, Northwestern University, Evanston, Illinois 60201, U.S.A.

(Received 5 August 1975; in revised form 13 September 1976)

\begin{abstract}
Low-frequency horizontally oriented gratings presented with an $180^{\circ}$ vertical interocular phase difference are detected in visual masking noise with nearly one log unit less contrast than gratings with no interocular phase difference. The difference in detectability, the Binocular MaskingLevel Difference (BMLD), does not depend on differences in the temporal characteristics of the signal and noise gratings. Although the BMLD is larger with narrowband noise than with broadband noise, it was not sufficiently larger to support the notion that the BMLD is based solely on interocular phase cues.
\end{abstract}

\section{INTRODUCTION}

Henning and Hertz (1973) used a very narrow band of spatial frequencies (one-dimensional visual noise) to mask a sinusoidal grating. Very nearly identical samples of the visual noise were presented to each eye, and the subjects fused the patterns to perceive a single $8.5^{\circ}$ square field in the plane of the display. The grating to be detected-the signal grating - was presented against this fuscd noise background in one of two conditions: either the signal grating, like the noise, was identical in the fields of both eyes, or the signal grating was $180^{\circ}$ out of phase between the fields, that is, the light bars of the grating in one field were, in the fused image, superimposed on the dark bars of the other field. Gratings of low spatial frequency were detected at much lower contrasts when they were presented $180^{\circ}$ out of phase than when they were presented in phase. The difference in detectability is analogous to the auditory binaural masking level difference (Durlach, 1972; Jeffress, 1972); in noise that is identical at both ears a tone is more audible when it is $180^{\circ}$ out of phase at the ears than when it is in the same phase at both ears.

The visual noise in the previous paper was derived from a noise wave-form that varied slowly during each observation interval. Thus the visual noise varied slowly in contrast and phase too. The signal grating, on the other hand, was fixed in contrast and phase and did not vary, even from interval to interval.

The experiments reported here extend our earlier findings to include noise that did not change throughout an observation interval, and broadband as well as narrowband visual noise.

\footnotetext{
${ }^{1}$ D.C.I.E.M. Research Paper 73-RP-97 6

2 The authors are the observers: observer 1 is $\mathrm{BGH}$, observer 2 is GBH.
}

\section{PROCEDURE}

A standard two-alternative temporal forced-choice grating detection task was used with horizontal signal and noise gratings. Each trial consisted of a brief warning interval followed by two $1-\mathrm{sec}$ observation intervals in only one of which a signal grating was presented. The observation intervals were separated by a $600 \mathrm{msec}$ pause. All intervals, including an answer interval, were marked for the obscrvers by bursts of sound. A new trial began every $4.5 \mathrm{sec}$ and 50 trials with one set of conditions were run at a time. The observers ${ }^{2}$ were required to say which observation interval had contained the signal and were informed, after responding, whether they were correct. The signal was always present in one or the other of the observation intervals and had probability 0.5 of being in the first interval on each trial.

The stimuli were generated, using the technique described by Campbell and Green (1965), on a HewlettPackard $1300 X-Y$ display which was masked to produce two separate visual fields each with an $8.5^{\circ}$ square aperture in a black surround. A matte black septum extended from the display to the observer so that the left field was seen by the left eye only and the right by the right. Prisms were used to allow the observer to fuse the two fields easily and see a single $8.5^{\circ}$ square aperture. No fixation marks were used.

Both signal and noise were generated from a computer. They were stored in digital form, subsequently read out through separate digital-to-analogue converters (DACs), and finally led through a suitable mixing network to the $Z$-axis of the display. A third DAC provided a synchronizing pulse for the $X$-sweep of the display. Both the signal and noise were turned on only during the observation intervals and caused no change in the mean luminance of the display.

The number of values of each wave-form sufficient to fill one field was produced by the computer; then, 
for the noise gratings, this same sample was reproduced to fill the other field so that the noise patterns were identical in the fields for each eye. (The septum masked the transient produced between the two fields.) The signal grating was produced in the same way when presented in identical phase in each field. When the signal was presented $180^{\circ}$ out of phase the grating for the right eye was simply shifted by half the spatial period of the grating without, of course, moving the field.

The noise comprised either a broad or a narrow band of spatial frequencies. The broadband noise contained all frequencies below $7.8 \mathrm{c} / \mathrm{deg}$, and had a mean noise contrast within that band of $7.6 \%$ per $\mathrm{c} / \mathrm{deg}$. This noise grating appeared to the observers as a stationary and irregular pattern of light and dark horizontal bars. The bandwidth of the narrowhand noise, which was centered on the signal frequency, was $0.003 \mathrm{c} / \mathrm{deg}$. Its mean contrast ${ }^{3}$ was $46.25 \%$. The narrowband noise looked like a sinusoidal grating with the same spatial frequency as the signal.

Like the signal, the noise gratings did not change within an observation interval. However, different samples of noise were chosen from observation interval to observation interval. This meant that both the phase and the contrast of the noise gratings varied from observation interval to observation interval. Both signal and noise were turned on and off without any temporal shaping and appeared only during the observation intervals.

The signal could be either identical in both ficlds or $180^{\circ}$ out of phase. The inversion was accomplished by switching an analogue inverter into the signal channel when the $X$-sweep was between the two fields.

The percentage of correct responses at various signal contrasts was determined with signal gratings of 0.46 and $1.85 \mathrm{c} / \mathrm{deg}$, for both in- and out-of-phase gratings, in the presence of both broad- and narrowband noise. At least four separate signal contrasts were used in each condition for each observer so that the slope of the function relating the percentage of correct responses to signal contrast might be roughly determined. The points above and below $75 \%$ correct responses were based on 200 observations for each observer, but the more extreme points each represent only 100 observations.

\section{RESULTS AND DISCUSSION}

Log signal contrast was approximately linearly related to the percentage of correct responses in the range $60-90 \%$ correct and the slopes of the psychometric function were roughly equal for all experi-

\footnotetext{
${ }^{3}$ There are a number of ways to describe the mean contrast of a visual noise. In describing the narrowband noise, we treated the noise grating as a sinusoidal grating varying in contrast and phase. The contrast is a Rayleigh distributed random variable and we have used the mean of the Rayleigh distribution as the mean noise contract. On the other hand, we have described the broadband noise in terms of the mean noise contrast per $\mathrm{c} / \mathrm{deg}$. This is perhaps a more appropriate description of the visual noise, but, if used in describing narrowband noise of bandwidth less than $1 \mathrm{c} / \mathrm{deg}$, leads to confusingly large mean contrasts.
}

ments. Thus we can reasonably compare our various conditions in terms of the signal contrast required for $75 \%$ correct responses. We shall call the contrast corresponding to $75 \%$ correct responses the masked contrast threshold. We have called the logarithm of the ratio of the masked contrast threshold for inphase signals to the masked contrast threshold for out-of-phase signals (a difference of logarithms) the Binocular Masking-Level Difference, or BMLD (Henning and Hertz, 1973) by analogy with the similar auditory effect. Figure 1 shows the BMLD in narrowband noise as a function of the spatial frequency of the signal grating. We have included data from our previous study, also for horizontal gratings and also detected in narrowband noise for comparison (closed symbols). The noise in the previous study varied slowly during an ohservation interval; the noise in the present study did not. The data indicate that the magnitude of the BMLD (approx $0.8 \mathrm{log}$ units at $0.46 \mathrm{c} / \mathrm{deg}$ ) was not erucially dependent on whether the contrast and phase of the masking noise varied within an observation interval; in both experiments low frequency signals presented out-of-phase in the two fields were detected with nearly an order of magnitude less contrast than in-phase signals. The results suggest that time-dependent cues are not particularly important determinants of the interocular phase effect.

Figure 2 shows the BMLD, again for horizontal gratings, measured for two signal frequencies in broadband noise. (The solid line connects the average of the data points at each frequency from Fig. 1 for comparison.) At the lower spatial frequency, broadband noise produced a smaller BMLD than narrowband noise, but the two types of noise produced a similar BMLD at the higher spatial frequency.

Although Figs. 1 and 2 make it clear that the BMLD may be produced with either broad- or narrowband noise and is not crucially dependent on differences in the temporal properties of the signal and noise, it is also important to consider the functions relating masked contrast threshold and spatial frequency in each condition of signal phase the func-

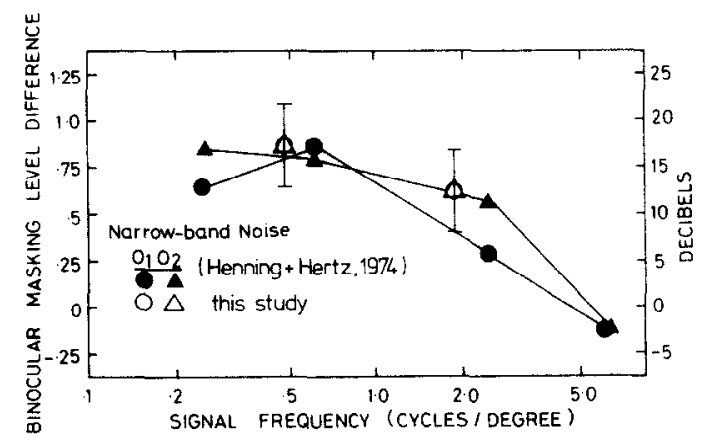

Fig. 1. The Binocular Masking-Level Difference for 0.46 and $1.85 \mathrm{c} / \mathrm{deg}$ sinusoidal gratings detected in stationary narrowband visual noise centered on the signal frequency (open symbols). The equivalent in decibels is shown on the right-hand co-ordinate. Data for the same two observers obtained with moving noise at other frequencies (closed symbols) arc included for comparison (Henning and Hertz, 1973). The vertical bars through the data point show a 2 S.D. range about the mean ratio. 


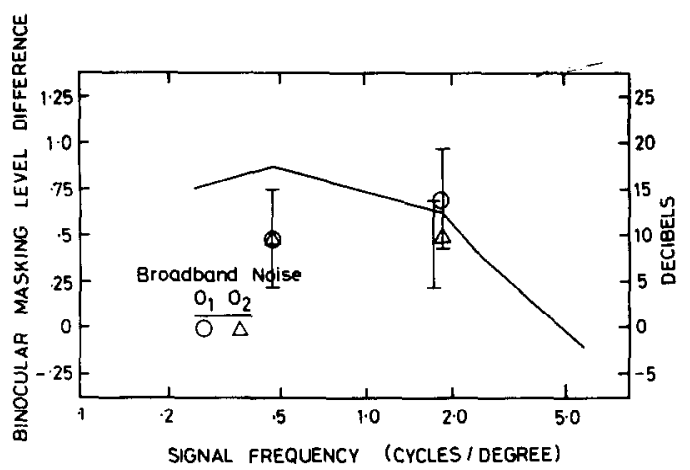

Fig. 2. The Binocular Masking-Level Difference for sinusoidal gratings detected in stationary broadband visual noise for two different spatial frequencies. The solid line shows average performance of the same observers detecting the same signal ratings in narrowband visual noise (from Fig. 1), as a comparison. The vertical bars through the data point show a 2 S.D. range about the mean ratio.

tions whose ratio determines the BMLD-because these functions may vary even though their ratio does not.

Figure 3a shows the masked contrast threshold for a signal in narrowband noise as a function of spatial frequency. Data from the broadband noise experiment are shown on the same co-ordinates in Fig. 3b, scaled to take into account the different noise levels in the two experiments. ${ }^{4}$

First, compare the in-phase conditions in Figs. 3a and $3 \mathrm{~b}$-the symbols without the stroke. Note that in noise of either bandwith, the signal frequency had little effect on detectability. This is consistent with the findings of Coltman and Anderson (1960) who found that signal frequency had negligible effect on the detectability of vertical gratings in "snow"broadband noise of all orientations. We should like, however, to compare the masked contrast thresholds for in-phase signals at given frequencies in the broadand narrowband noise cases, but it is difficult to determine the appropriate mode of comparison because the appropriate way to equate the two types of noise depends on the detection mechanism that our observers used.

For example, Fig. 3a shows the actual contrast necessary for detecting the signal in narrowband noise. The contrasts in Fig. 3b, on the other hand, have all been increased by a factor of 1.73 to equate

\footnotetext{
${ }^{4}$ Scaling of signal contrasts is appropriate, for in-phase signals at least, because changes in noise level produce proportional changes in the contrast of just-detectable signals (Stromeyer and Julesz, 1972).

${ }^{5}$ The luminance pattern in our display, $L(s)$, may be written in the form
}

$$
L(s)=\bar{L}[1+c f(s)] .
$$

where $\bar{L}$ is the mean luminance, $f(s)$ is the normalized spatially varying component and $c$ is a scaling factor that determines the contrast of the pattern. After Carter and Henning (1971), we take the grating "power" to be $c^{2} f^{2}(s)$ and the grating "energy", $E$, as given by

$$
E=\int c^{2} f^{2}(s) \mathrm{d} s,
$$

where the integral is taken over the extent of the grating. the total noise power in the broad- and narrowband masking stimuli. ${ }^{5}$ [The broad- and narrowband noise in our experiment had almost identical root-meansquare contrast simply because we wanted to ensure: (a) that every signal, i.e. even the ones which were totally undetectable in the noise, could be detected without error in the absence of noise, and (b) that the sum of the signal and noise rarely exceeded the linear dynamic range of our display.]

Now it is known that the relation between mean noise contrast and masked threshold contrast is linearly proportional within the limits of contrast used in our experiments (Stromeyer and Julesz, 1972). We can thus legitimately make a different and potentially interesting comparison between the effects of broad- and narrowband noise when they have been equated for noise power per cycle per degree. This comparison is interesting because it allows us to estimate the effective bandwidth of the spatial frequency channels hypothesized by Campbell and Robson (1968). Provided we assume that the ratio of signal to noise power within a channel is the determining factor in detection, the channel widths at each frequency are given simply as the ratio of the square of the threshold contrasts in the broad- and narrowband noise conditions. Calculation then suggests that the effective bandwidths are $1.1 \mathrm{c} / \mathrm{deg}$ at $0.46 \mathrm{c} / \mathrm{deg}$ and $1.6 \mathrm{c} / \mathrm{deg}$ at $1.85 \mathrm{c} / \mathrm{deg}$.

Comparison of the effects of different noise bandwidths on the detectability of out-of-phase signals must be made with caution; the effect of noise level on detectability in this case has not been determined,

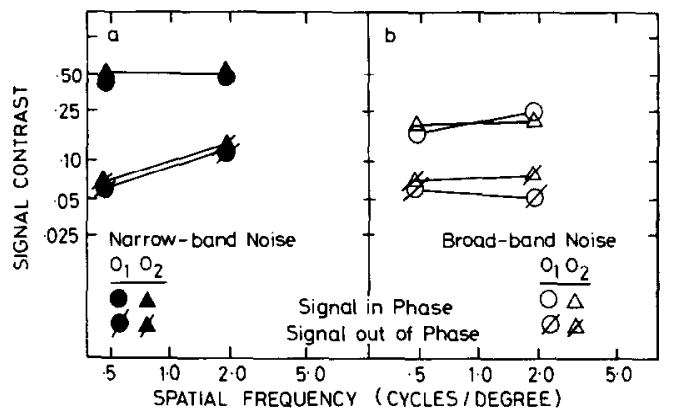

Fig. 3a. The contrast corresponding to $75 \%$ correct detection of the signal gratings (masked contrast threshold) in narrowband visual noise centered on the signal frequency as a function of signal contrast. The mean noise contrast (see footnote 3) was $46.25 \%$. The symbols with a stroke through them represent conditions in which the signal gratings were $180^{\circ}$ out of phase in the two visual fields; symbols with no stroke, conditions with in-phase signals. The standard deviation is only slightly larger than the data symbols.

Fig. 3b. The contrast corresponding to $75 \%$ correct detection of the signal gratings (masked contrast threshold) in narrowband visual noise centered on the signal frequency as a function of signal contrast. The thresholds have been scaled to adjust for differences in total noise power between this broad- and the narrowband maskers (see footnote 4). The mean noise contrast was $7.6 \% \mathrm{c} / \mathrm{deg}$. The symbols with a stroke through them represent conditions in which the signal gratings were $180^{\circ}$ out of phase in the two visual fields; symbols with no stroke, conditions with in-phase signals. The standard deviation is only slightly larger than the data symbols. 
and noise level is known to affect the masking level difference found in hearing (Durlach, 1972). However, if we make the comparison when the noise power per c/deg for two bandwidths are equated we find that our horizontal out-of-phase gratings are detected more readily in narrow than in broadband noise. This finding might be anticipated on the assumption that out-of-phase signals are detected on the basis of interocular phase differences. In masking experiments using noise, interocular phase is a random variable whose distribution depends only on a quantity we shall call Egiven by

$$
\xi=C_{s}\left(N_{0} \cdot W\right)^{12}
$$

where $C_{s}$ is the signal contrast, $N_{0}$ the noise contrast/ $\mathrm{c} / \mathrm{deg}$, and $W$ is the effective bandwidth of the noise in c/deg (Henning, 1973). If observers base their decisions on interocular phase differences, then, whenever $\xi$ is constant, a constant level of performance should result. Equation (1) shows that to keep $\xi$ constant $C_{s}^{2}$ should decrease in proportion as $W$ increases, and thus the contrast at which an out-of-phase signal is detected in noise should decrease with decreasing noise bandwidth. Using equation (1) and the assumptions just mentioned, we can derive another estimate for the width of spatial frequency channels; the bandwidth is $1.6 \mathrm{c} / \mathrm{deg}$ at $1.85 \mathrm{c} / \mathrm{deg}$ but is $7 \mathrm{c} / \mathrm{deg}$ at $0.46 \mathrm{c} / \mathrm{deg}$. The estimate for the higher frequency signal is reasonable; on the other hand, an effective bandwidth of $7 \mathrm{c} / \mathrm{deg}$ at 0.46 seems highly unlikely and casts doubt on the assumption that observers base their decisions solely on interocular phase differences.

The Binocular Masking-Level Difference must arise from interocular phase and contrast dependent cues produced when an out-of-phase signal is added to the masking noise-these would seem to be the only additional cues available in the out-of-phase case.
However, the experimental results just described indicate that it is unlikely that interocular phase differences alone provide adequate cues for the detection of the out-of-phase grating; interocular contrast differences must produce significant cues for the BMLD.

Acknowledgements - We are grateful to Professor I. Nachmias and to Dr. J. G. Robson for their helpful criticism of this paper. The research was supported, in part. by grants from the S.R.C. and N.I.H.

\section{REFERENCES}

Campbell F. W. and Green D. G. (1965) Optical and retinal factors affecting visual resolution. J. Physiol, Lond. 181, 576-593.

Campbell F. W. and Robson J. G. (1968) Application of Fourier analysis to the visibility of gratings. $J$. Physiol. Lond. 197, 551-556.

Carter B. E. and Henning G. B. (1971) The detection of gratings in narrowband visual noise. I. Physiol., Iond. 219, 355-365.

Coltman J. W. and Anderson A. E. (1960) Noise limitations to resolving power in electronic imaging. Pror. Instn Radio Engrs May, 858-865.

Durlach N. I. (1972) Binaural signal detection: equalization and cancellation theory. In Foundations of Modern Auditory Theory II (Edited by Tobias J. V.). Academic Press, New York.

Henning G. B. (1973) Effect of interaural phase on frequency and amplitude discrimination. J. acoust. Soc. Am. 54, 1160-1178.

Henning G. B. and Hertz B. G. (1973) Binocular masking level differences in sinusoidal grating detection. Vision Res. 13, 2455-2463.

Jeffress L. A. (1972) Binaural signal detection: vector theory. In Foundations of Modern Auditory Theory II (Edited by Tobias J. V.). Academic Press, New York.

Stromeyer C. F. and Julesz B. (1972) Spatial frequency masking in vision: critical bands and spread of masking. J. opt. Soc. Am. 62, 1221-1232. 